\title{
Inter-operator variability in the sensitivity of sentinel lymph node biopsy for melanoma
}

Conrad Harrison ${ }^{1}$, Jeremy N Rodrigues ${ }^{2}$, Oliver Cassell ${ }^{1}$

1. Blenheim Head and Neck Unit, Churchill Hospital, Old Road, Headington, Oxford OX3 7LE

2. The Nuffield Department of Orthopaedics, Rheumatology and Musculoskeletal Sciences, University of Oxford, Nuffield Orthopaedic Centre, Windmill Road, Headington, Oxford, OX3 7HE, UK

Corresponding author:

Conrad Harrison

Medical Sciences Office, University of Oxford,

John Radcliffe Hospital,

Headley Way,

Headington,

Oxford

OX3 9DU

Telephone: 07817621787

E-mail: Conrad.harrison@medsci.ox.ac.uk

Word Count: 713 


\section{Abstract}

Introduction and aims: Plastic surgery trainees, in some circumstances, can progress to consultant level having performed only 15 generic lymph node surgeries, with no mention specifically of sentinel lymph node biopsy (SLNB) on the training log. The majority of SLNBs carried out for melanoma at our centre since 1999 have been performed by eight surgeons, six of whom had been formally trained and mentored in our unit or previously completed skin cancer fellowships. Two surgeons started performing the procedure without formal training or specialist fellowships. We analysed the first 40 cases performed by each consultant hypothesising that those with more extensive training would achieve higher sensitivities.

Methods: Using our centre's prospectively collected data set we studied 320 procedures. Sensitivities were calculated and compared between surgeons with less extensive and more extensive training.

Results: The two surgeons without formal training had a combined sensitivity of $85 \%$ ( 80 cases, 17 positive results, 3 false negative results). The six surgeons who had been practising after extensive training had a combined sensitivity of $94 \%$ ( 240 cases, 44 positive results, 3 false negative results). SLNBs in the head and neck region accounted for $17 \%$ of total cases, but $50 \%$ of false positive results.

Conclusions: There is likely to be a learning curve for this operation, and in the era of adjuvant therapy a false negative result may affect survival. Training requirements may not be enough to ensure consistency amongst newly qualified plastic surgeons. We advocate a similar level of experience to that required of breast surgeons before performing the procedure independently. 
Dear Sirs,

Following a diagnosis of melanoma, a negative sentinel lymph node biopsy (SLNB) is reassuring, but a cohort of patients will have false negative results and be left with residual cancer in the sampled basin. With the dawn of adjuvant treatment in sentinel node positive patients (Stage IIla) the accuracy of the SLNB result will influence survival. False negative results are usually defined as a nodal recurrence following a negative SLNB in the same basin. Rates of false negative results vary in the literature depending on the calculation used, some authors choosing to divide the number of false negatives by total sample size, total number of negative biopsies or the sum of false negatives and true positives. Terms such as sensitivity may avoid confusion, and this ranges from $79-91 \%$ (1).

To ensure accuracy in this diagnostic procedure, trainee breast surgeons in the UK must complete 70 axillary SLNBs under supervision before gaining their certificate of completion of training (2). This target is supported by evidence that breast surgeons experience a learning curve when starting to perform this operation, with accuracy improving over their first 20 cases (3). On the other hand, plastic surgery trainees, in some circumstances, can progress to consultant level having performed only 15 generic lymph node surgeries, with SLNB not specifically stipulated on the Joint Committee on Surgical Training (JCST) certification guidelines (2). The accuracy of SLNB for breast cancer has been shown to be surgeon-dependent (4), and in this study we aimed to investigate whether there was a surgeon-specific variability in the sensitivity of SLNB for melanoma amongst new consultants.

Our centre has a large skin cancer unit and was one of the first in the country to introduce SLNB for melanoma. Today, our independent operators are expected to have well exceeded the indicative numbers for lymph node surgery set out by the JCST. The majority of SLNBs carried out at our centre since 1999 have been performed by eight surgeons, six of whom had been formally trained and mentored in our unit or previously completed skin cancer fellowships. Two surgeons undertook sentinel node biopsy without formal mentored training.

We analysed the first 40 cases performed by each consultant with a null hypothesis that surgeons perform the procedure without inter-operator differences in sensitivity and an alternative hypothesis that those with more extensive training would achieve higher sensitivities. Sensitivity was defined as the number of positive biopsies divided by the sum of the number of positive biopsies and false negatives. False negatives were defined as a clinical nodal recurrence following a negative SLNB in the same nodal basin.

We studied 320 SLNBs performed on 297 patients (with some participants having the procedure performed in multiple nodal basins). Of these 320,61 were positive and 259 were recorded as negative. After a mean follow up of 6.6 years, six cases were found to be false negatives. The two surgeons who had started practising without formal training had a combined sensitivity of $85 \%$ ( 80 cases, 17 positive results, 3 false negative results). The six surgeons who had been practising after extensive training had a combined sensitivity of $94 \%$ ( 240 cases, 44 positive results, 3 false negative results). SLNBs in the head and neck region accounted for $17 \%$ of total cases, but $50 \%$ of false positive results. With a total of six false negative results, further multivariate statistical analysis was not appropriate, as 10 false negatives per covariate is recommended for such analyses (5).

In this study, the two surgeons without formal training attained as many false negative results in their first 40 cases as the other six surgeons combined. There is likely to be a learning curve for this operation, as described in SLNB for breast cancer (3). Due to the importance of the SLNB result in managing patients with melanoma we suggest that the JCST should stipulate sentinel node biopsy as a specific procedure on the certification checklist. For surgeons specialising in skin cancer surgery, 
we advocate a similar level of experience to that required of breast surgeons before performing the procedure independently.

Head and neck sentinel node biopsy in our unit is now only carried out by specialist head and neck plastic surgeons, specifically trained in this area, who form part of skin and head and neck multidisciplinary teams.

Conrad Harrison ${ }^{1}$

Jeremy N Rodrigues ${ }^{2}$

Oliver Cassell ${ }^{1}$

1. Blenheim Head and Neck Unit, Churchill Hospital, Old Road, Headington, Oxford OX3 7LE

2. The Nuffield Department of Orthopaedics, Rheumatology and Musculoskeletal Sciences, University of Oxford, Nuffield Orthopaedic Centre, Windmill Road, Headington, Oxford, OX3 7HE, UK

\section{Funding}

Nil specific. JNR is funded by an NIHR Postdoctoral Fellowship. The views expressed are those of the authors and not necessarily those of the NIHR, NHS or Department of Health.

\section{Conflict of Interests}

Nil

\section{References}

1. Testori A, De Salvo GL, Montesco MC, et al. Clinical considerations on sentinel node biopsy in melanoma from an Italian multicentric study on 1,313 patients (SOLISM-IMI). Ann Surg Oncol 2009;16(7):2018-27.

2. Joint Committee on Surgical Training. Certification guidelines and checklists. https://www.jcst.org/quality-assurance/certification-guidelines-and-checklists/. [Accessibility verified July 18, 2018]

3. McMasters KM, Wong SL, Chao C, et al. Defining the optimal surgeon experience for breast cancer sentinel lymph node biopsy: a model for implementation of new surgical techniques. Ann Surg 2001;234(3):292-300

4. Cody HS $3^{\text {rd }}$. Sentinel lymph node mapping in breast cancer. Breast Cancer 1999;6(1):13-22

5. Peduzzi $P$, Concato J, Kemper $E$, et al. A simulation study of the number of events per variable in logistic regression analysis. J Clin Epidemiol. 1996, 49: 1373-9. 